\title{
Stem cell therapy for neurological disorders
}

\author{
M Alessandrini, ${ }^{1} \mathrm{PhD}$; O Preynat-Seauve, ${ }^{2,3} \mathrm{PhD}$, PharmD, PD; K de Bruin, ${ }^{4}$ BEng, BEng (Hons), MEng; M S Pepper, ${ }^{4} \mathrm{MB}$ ChB, PhD, MD \\ ${ }^{1}$ Department of Pathology and Immunology, Faculty of Medicine, University of Geneva, Switzerland \\ ${ }^{2}$ Laboratory of Therapy and Stem Cells, Department of Diagnostics, Laboratory Medicine and Pathology, Geneva University Hospitals, \\ Switzerland \\ ${ }^{3}$ Department of Medical Specialties of Internal Medicine, Faculty of Medicine, University of Geneva, Switzerland \\ ${ }^{4}$ Institute for Cellular and Molecular Medicine, Department of Immunology; and SAMRC Extramural Unit for Stem Cell Research and Therapy, \\ Faculty of Health Sciences, University of Pretoria, South Africa
}

Corresponding author: M Alessandrini (marco.alessandrini@unige.ch)

\begin{abstract}
Neurological disease encompasses a diverse group of disorders of the central and peripheral nervous systems, which collectively are the leading cause of disease burden globally. The scope of treatment options for neurological disease is limited, and drug approval rates for improved treatments remain poor when compared with other therapeutic areas. Stem cell therapy provides hope for many patients, but should be tempered with the realisation that the scientific and medical communities are still to fully unravel the complexities of stem cell biology, and to provide satisfactory data that support the rational, evidence-based application of these cells from a therapeutic perspective. We provide an overview of the application of stem cells in neurological disease, starting with basic principles, and extending these to describe the clinical trial landscape and progress made over the last decade. Many forms of stem cell therapy exist, including the use of neural, haematopoietic and mesenchymal stem cells. Cell therapies derived from differentiated embryonic stem cells and induced pluripotent stem cells are also starting to feature prominently. Over 200 clinical studies applying various stem cell approaches to treat neurological disease have been registered to date (Clinicaltrials.gov), the majority of which are for multiple sclerosis, stroke and spinal cord injuries. In total, we identified 17 neurological indications in clinical stage development. Few studies have progressed into large, pivotal investigations with randomised clinical trial designs. Results from such studies will be essential for approval and application as mainstream treatments in the future.
\end{abstract}

S Afr Med J 2019;109(8 Suppl 1):S71-S78. https://doi.org/10.7196/SAMJ.2019.v109i8b.14009

Disorders of the central and peripheral nervous systems are diverse and heterogeneous. Hundreds of millions of people worldwide are affected by neurological disorders. Overall, these are the leading cause of disability-adjusted life years (DALYs), making up 10.2\% of the total disease burden worldwide in 2015. Neurological disorders are second only to cardiovascular disease regarding causes of death - $16.8 \%$ of deaths in 2015 were due to neurological conditions. ${ }^{[1]}$ It has previously been reported that the annual cost of managing neurological disease in the USA alone is close to USD800 billion. ${ }^{[2]}$ Despite this health burden, the treatment landscape is limited, with few curative options available and poor drug approval rates for potential new medications. ${ }^{[3]}$

Stem cells have in many respects revolutionised the way in which we explore and treat disease. However, with the exception of haematopoietic stem cell (HSC) transplantation, the stem cell hype is yet to live up to its promise concerning treatment and cure of disease. The reasons for this are manifold, but primarily because we have still to understand the complete modus operandi of the various stem cell preparations and their clinical implications. Recently, Japanese authorities cleared a mesenchymal stem cell (MSC) product for treating spinal cord injuries. Although concerns over the approval have been raised by the global scientific community, ${ }^{[4]}$ this is the first and only stem cell therapy approved for treating a neurological condition. Nonetheless, there is encouraging evidence to suggest that stem cell therapy as a whole holds promise for drug approvals in this area in the future. We provide a high-level perspective of where progress has been made with respect to the use of stem cells for treating neurological disease.

\section{Stem cells}

Stem cells provide the building blocks for every organ in the body. They have the unique ability to divide asymmetrically, and to differentiate into the various cell types of the body while simultaneously replicating to maintain a stem cell lineage. Stem cells are present in almost every human tissue - in embryos, they differentiate into all the tissues and organs of the body, and in fully developed humans they provide a renewal capacity in most organs. Different forms of stem cells exist, each with a varying 'potency' (Fig. 1). Potency refers to the ability of a stem cell to replicate and differentiate into different cell types. Totipotent and pluripotent stem cells have the highest potency, and are obtained from the pre-embryonic stages of development. Blastomeres that arise from a fertilised ovum (zygote) are totipotent stem cells and have the potential to differentiate into all organs of the body, including the placenta. A cluster of blastomeres goes on to form a blastocyst, which has a hollow cavity and an inner cell mass of embryonic stem cells (ESCs). These stem cells are pluripotent in nature and, relative to totipotent stem cells, have lost the ability to differentiate into the placenta while maintaining their ability to form all organs and tissues of the body. A blastocyst develops into a gastrula wherein the three germ layers have started to form, followed by the development of an embryo.

Pluripotent stem cells differentiate into multipotent stem cells, including HSCs, MSCs and neural stem cells (NSCs). These cells are committed to further differentiate into cells of a given organ or system, e.g. HSCs differentiate into cells of the blood and immune systems, and NSCs into cells of the brain and nervous 


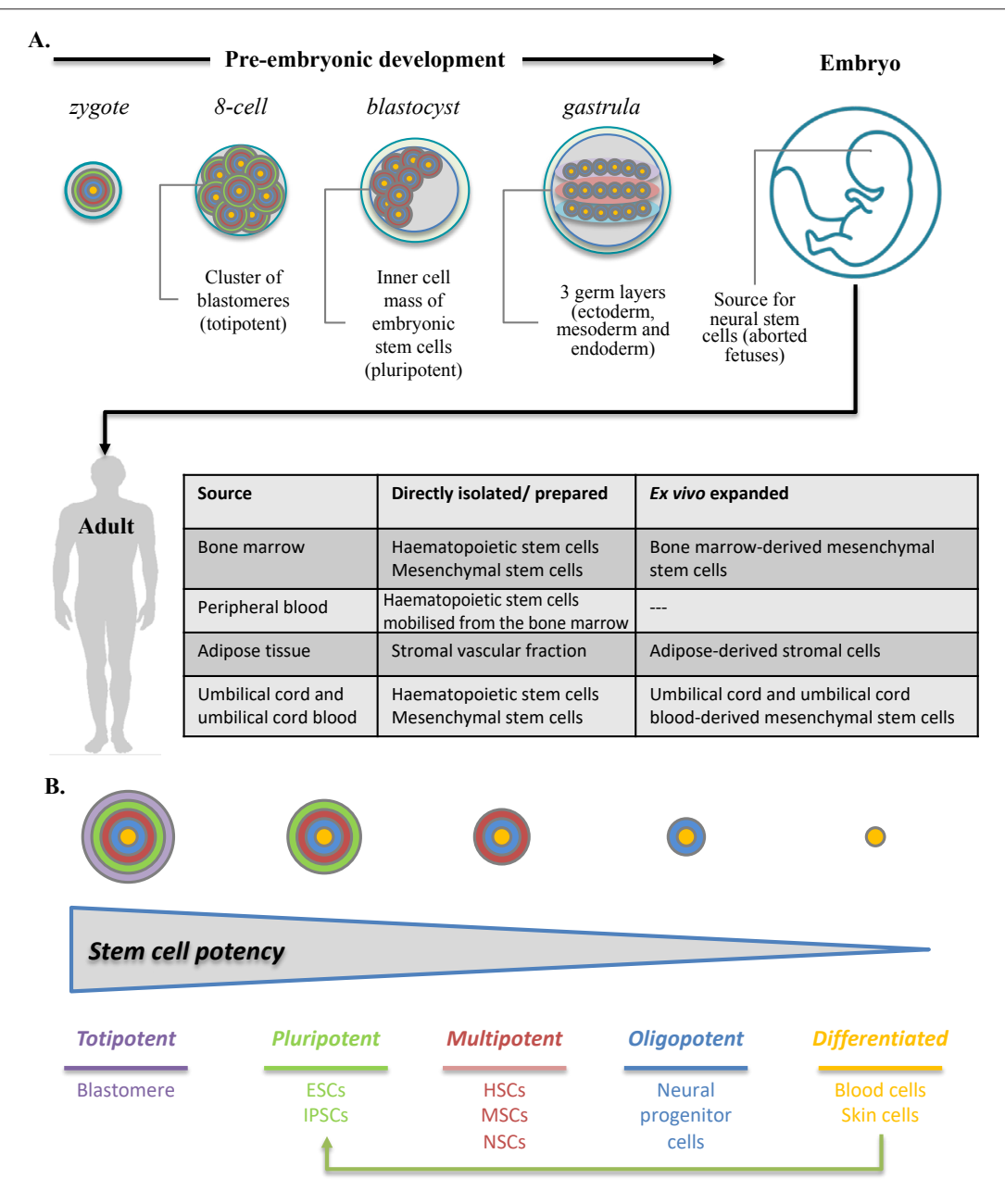

Fig. 1. Schematic summary of stem cell sources and potency. (A) Totipotent (blastomeres) and pluripotent stem cells (embryonic stem cells (ESCs)) are obtained from pre-embryonic tissue. Multipotent neural stem cells are harvested from aborted fetuses, while haematopoietic and mesenchymal stem cells (including adipose stromal cells) are collected from different sources in adults. (B) Diagram illustrating the potency of the different stem cell types. Multipotent stem cells differentiate into oligopotent stem cells, such as lineage-specific progenitor cells, which ultimately differentiate into fully committed cells, such as blood cells or skin cells. Induced pluripotent stem cells (iPSCs) can be derived from various types of fully committed/differentiated cells. (HSCs = haematopoietic stem cells; MSCs = mesenchymal stem cells; NSCs = neural stem cells.)

Image of fetus created by Laymik from the Noun Project.

system. Multipotent stem cells differentiate into progenitor cells that are oligo- and unipotent, which are further committed to a limited number of cell types. Until recently, stem cell differentiation was considered unidirectional; however, by introducing a defined number of factors into fully differentiated cells (e.g. skin or blood cells) in a laboratory setting, one is able to derive a cell type which once again has pluripotent capacity - these are referred to as induced pluripotent stem cells (iPSCs).

\section{Stem cell therapy for neurological disorders}

In principle, for a stem cell therapy to be therapy for treating neurological disorders. However, since they are located deep within the adult brain, NSCs are not accessible for harvesting and hence for autologous therapeutic applications. They are therefore procured from aborted fetuses (i.e. aborted for medical or non-medical reasons) and prepared for allogeneic transplantation purposes. Given the practical and ethical limitations of this scenario, in addition to the risks that transplanted cells might be rejected by the recipient's immune system ${ }^{[5]}$ or that they may be tumorigenic (owing to their heightened proliferative capacity), there has been an appreciable increase in the need for an alternative source. With recent advances in our understanding of stem cell biology, it is possible to differentiate pluripotent stem cells (both ESCs and iPSCs) into neural progenitor or NSC-like cells for therapeutic purposes. Protocols for differentiating bone marrow-derived MSCs into NSC-like cells have also been developed and translated for clinical application in patients with multiple sclerosis. ${ }^{[6]}$ Both forms of derivation (from fetal tissue and differentiated) are being investigated in clinical studies to treat several neurological conditions. Given their high proliferative capacity and risk for tumour formation, pluripotent stem cells are not used directly for treatment purposes, but rather are differentiated into the desired fully differentiated cell type. HSCs and MSCs are also being explored in clinical studies to investigate their potential for treating various neurological disorders.

To better understand the treatment landscape of stem cell therapies for brain disease, we extracted data from the Clinicaltrials.gov website to identify and investigate which therapies have progressed into clinical development stages. A database of registered clinical trials was created based on data outputs using the following search terms: 'embryonic stem cells', 'induced pluripotent stem cells', 'neural stem cells', 'mesenchymal stem cells', 'mesenchymal stromal cells', 'bone marrow stromal cells', 'umbilical cord mesenchymal stem cells', 'adipose stem cells', 'adipose-derived regenerative cells' and 'stromal vascular fraction'. To capture trials using HSCs, we based our search on the use of 'haematopoietic stem cells' and 'umbilical cord blood' together with disease indications listed in one of our previous reports. ${ }^{[7]}$ The data were cleaned to harmonise terminology across all trials and categorised into therapeutic areas. Observational studies were excluded and all trials relating to neurological disease were selected and taken forward for analysis and interpretation. Relevant clinical 
Table 1. Advantages and disadvantages of the different stem cell types

\begin{tabular}{|c|c|c|}
\hline Stem cells & Advantages & Disadvantages \\
\hline \multirow[t]{3}{*}{ NSCs } & Prototype stem cells for treating neurological disease & $\begin{array}{l}\text { Limited resource with ethical implications around procurement } \\
\text { (if from aborted fetal tissue) }\end{array}$ \\
\hline & $\begin{array}{l}\text { NSC-like cells can be derived from other stem cell types } \\
\text { (pluripotent and multipotent) }\end{array}$ & $\begin{array}{l}\text { Poorly understood stem cell biology and least explored in clinical } \\
\text { studies }\end{array}$ \\
\hline & & Tumorigenic risks if derived from pluripotent stem cells \\
\hline \multirow[t]{3}{*}{ HSCs } & $\begin{array}{l}\text { Globally accepted form of treatment for haematological } \\
\text { conditions }\end{array}$ & Limited experience for use in neurological disease \\
\hline & $\begin{array}{l}\text { Well-established industry for harvesting and preparation } \\
\text { of clinical grade treatments }\end{array}$ & $\begin{array}{l}\text { Generally limited for use as an autologous therapy (requires } \\
\text { genetic matching of the donor and recipient if used as an } \\
\text { allogeneic treatment) }\end{array}$ \\
\hline & & $\begin{array}{l}\text { Poorly understood mechanism of action for treating certain } \\
\text { neurological conditions }\end{array}$ \\
\hline \multirow[t]{3}{*}{ MSCs } & Readily accessible resource and easily procured & Exploited by unregulated clinics globally \\
\hline & No need for genetic matching & $\begin{array}{l}\text { Poorly understood mechanism of action for treating certain } \\
\text { neurological conditions }\end{array}$ \\
\hline & $\begin{array}{l}\text { Most likely stem cell therapy to evolve into an off-the- } \\
\text { shelf allogenic product }\end{array}$ & \\
\hline
\end{tabular}

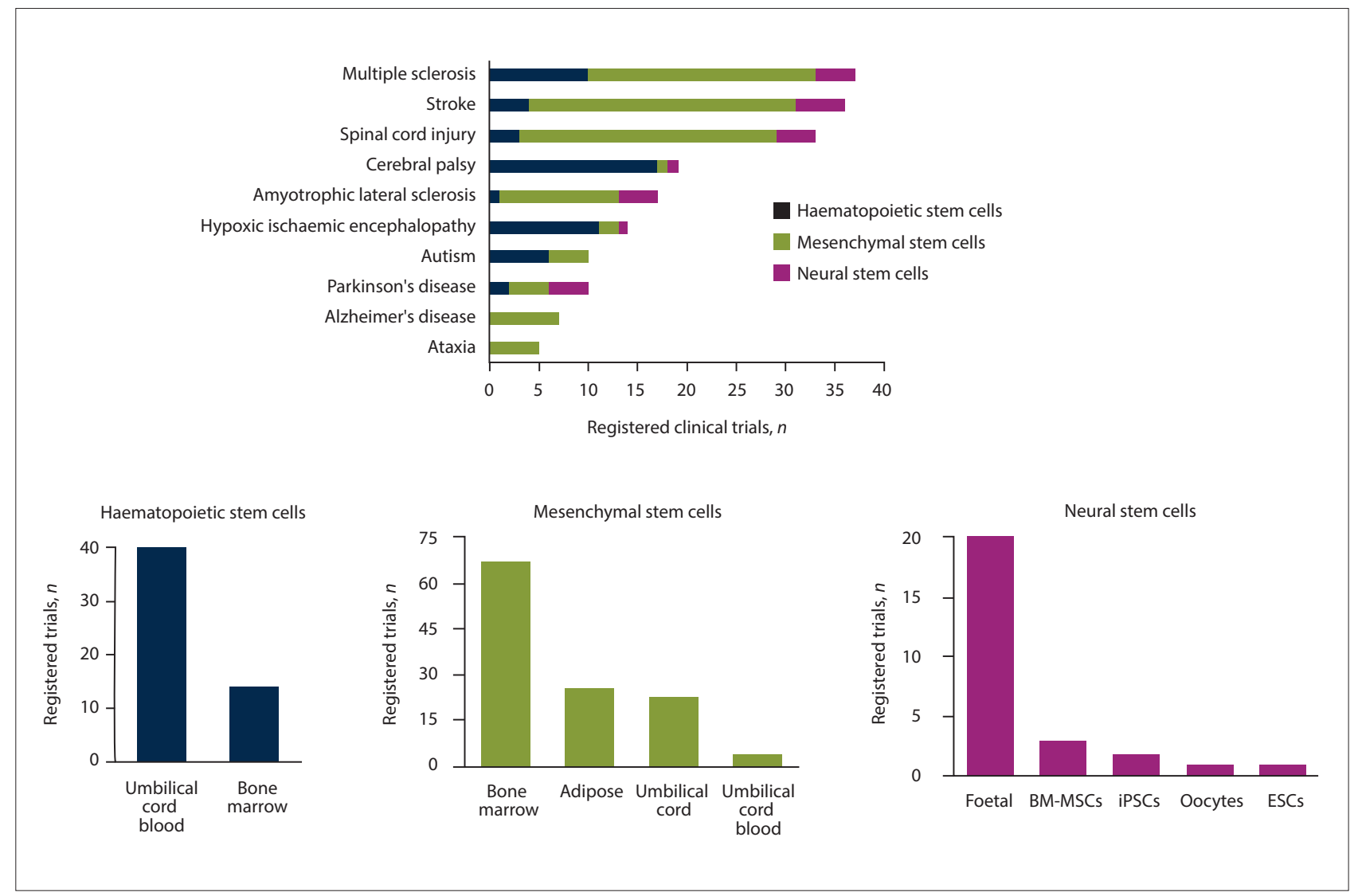

Fig. 2. Registered clinical trials for neurological conditions treated with stem cells. Registered studies $(\mathrm{N}=201)$ were exported from Clinicaltrials.gov. In order to capture a representative dataset, we expanded our searches to include (i) embryonic and induced pluripotent stem cells as a source for NSC-like cells; (ii) umbilical cord blood as a source for HSCs; and (iii) umbilical cord, umbilical cord blood, bone marrow and adipose for MSCs. The captured data were filtered to include only interventional studies for neurological diseases, and duplicate, terminated or withdrawn studies were excluded. Indications with less than 5 registered clinical studies include brain tumours $(\mathrm{n}=4)$, multiple system atrophy $(\mathrm{n}=2)$, diabetic neuropathy $(\mathrm{n}=2)$, cerebral adrenoleukodystrophy $(\mathrm{n}=1)$, epilepsy $(\mathrm{n}=1)$, mental retardation $(\mathrm{n}=1)$, progressive supranuclear palsy $(\mathrm{n}=1)$ and fibromyalgia $(\mathrm{n}=1) .(B M-M S C s=$ bone marrow-derived mesenchymal stem cells; ESCs = embryonic stem cells; iPSCs = induced pluripotent stem cells.) 
studies and disease indications were also searched online and in published literature via Pubmed and Google Scholar.

Fig. 2 provides an overview of globally registered clinical studies where stem cells have been applied for treating neurological disorders. Overall, we identified 201 clinical studies that were registered over the period of 1999 to 2018, of which more than half are indicated for the treatment of multiple sclerosis, stroke and spinal cord injury. MSCs are/were used in 120 studies, while HSCs and NSCs are/were applied in 54 and 27 trials, respectively. Ten stem cell therapies have progressed to advanced clinical stages (Phase II/III or III), including MSCs for spinal cord injury $(n=3)$, stroke $(n=2)$, cerebral palsy $(n=1)$ and diabetic neuropathy $(n=1)$; HSCs for multiple sclerosis $(n=2)$; and a single NSC treatment for Parkinson's disease. A more detailed perspective on the use of NSCs, HSCs and MSCs in this context is provided below.

\section{Neural stem cells}

NSCs are multipotent progenitors which have the ability to produce neurotrophic factors and/or differentiate into committed cell lineages of the central and peripheral nervous systems, including neurons and supporting glial cells (such as astrocytes and oligodendrocytes). In the adult brain, NSCs are limited to the hippocampus and play a role in supporting plasticity. Those found in the subventricular zone (SVZ) of the hippocampus are in contact with the cerebrospinal fluid, moving radially and differentiating to young neuroblasts. ${ }^{[8]}$ NSCs in the subgranular zone (SGZ) also move radially and differentiate to neuroblasts, but are referred to as radial glia-like NSCs. Neuroblasts go on to mature and differentiate into neurons. ${ }^{[9,10]}$ NSCs are abundant in fetal brains, most notably at very early stages of development. Once harvested from the fetal brain, they can be dissociated and grown in the laboratory as a monolayer of cells or as floating 'spheroids' that produce neurotrophic factors that can be differentiated into more defined neuronal lineages. ${ }^{[11}$

Pluripotent stem cells are increasingly becoming an important source for the generation of neural precursor cells. Using a variety of in vitro conditions, cells resembling NSCs (NSC-like) can be generated from human ESCs. These conditions include co-culturing with animal stromal cells (unknown stimuli), ${ }^{[12]}$ exposure to retinoic acid, ${ }^{[13]}$ and inhibition of bone morphogenetic protein (BMP) signalling. ${ }^{[14]}$ The recent development of iPSCs, which have also been shown to generate NSC-resembling cells, ${ }^{[15]}$ mitigates ethical concerns linked to the use of embryos and also introduces the possibility of autologous transplantation using patient-derived iPSCs. ${ }^{[16]}$

The rationale for therapeutic application of NSCs and NSClike cells is based on their ability to release neurotrophic factors and to differentiate into neural and glial cells, thereby promoting neurogenesis and replacing diseased or injured areas of the brain. To date, no NSC-based therapy has been approved for routine clinical use. We identified 27 registered clinical trials, of which two-thirds $(n=20)$ used fetal-derived NSCs for the treatment of neurological disease (Fig. 2). Most of the clinical studies using NSCs and NSC-like cells were for stroke $(n=5)$, while 4 studies each were for multiple sclerosis, spinal cord injury, amyotrophic lateral sclerosis, Parkinson's disease and brain tumours. For the latter, gene-modified NSCs are being applied (see below). Seven of the 27 studies using NSC-based therapies to treat neurological disease make use of NSC-like cells differentiated from bone marrow MSCs $(n=3)$, iPSCs $(n=2)$, ESCs $(n=1)$ and oocytes $(n=1)$.

Stroke occurs either as a result of impaired blood flow (ischaemic) or rupturing of blood vessels (haemorrhagic) in the brain, resulting in diminished oxygen and nutrient supply to neurons. Preclinical studies using NSCs to treat stroke have demonstrated that, in addition to stimulating neurogenesis, NSCs are capable of releasing angiogenic factors to promote local tissue regeneration. ${ }^{[17-19]}$ Four of the 5 clinical trials registered for stroke apply fetal-derived NSCs, of which 3 used gene-modified NSCs to create a conditionally immortalised cell line. Gene modification to express the recombinant protein, c-MycER ${ }^{\text {TAM }}$, allows for controlled expansion of a defined and homogeneous NSC cell line. ${ }^{[20]}$ In the completed Phase I and II studies (NCT01151124, NCT02117635), it was shown that these cells are well tolerated and the application is associated with improved neurological outcomes. ${ }^{[21]}$ A larger Phase IIb clinical study is ongoing (NCT03629275). The fourth study using fetal-derived NSCs is also still ongoing (NCT03296618), and notably, this same product (NSI566 ) is being tested in clinical studies for amyotrophic lateral sclerosis and spinal cord injury. In the most recently registered clinical study to be initiated in 2019, iPSC-derived NSC-like cells will be used to treat patients with haemorrhagic stroke (NCT03725865).

Multiple sclerosis is a neurodegenerative disorder characterised by inflammatory demyelination of neurons. Of the 4 registered clinical trials using NSCs to treat multiple sclerosis, 2 make use of fetalderived NSCs, while the other 2 apply bone marrow MSC-derived NSC-like cells. The fetal-derived NSC studies are still ongoing and no data have been reported. Recently reported findings from a Phase I clinical study (NCT01933802) showed that intrathecal administration of MSC-derived neural progenitor cells for patients with multiple sclerosis was safe and well tolerated ${ }^{[6]}$ Although only modest improvements were observed, the initial findings warrant further clinical development and initiation of a Phase II study (NCT03355365).

Of the 4 registered clinical studies using NSCs for spinal cord injury, 3 make use of fetal-derived NSCs, while the fourth uses NSClike cells differentiated from bone marrow MSCs. Data from one of these clinical studies have been reported which demonstratesthe safety of fetal-derived NSC transplantation (NSI-566), but with no significant improvement with respect to quality-of-life score. ${ }^{[22]}$ Improved secondary outcomes and encouraging preclinical data warrant testing in a larger dose escalation study. Although no trial of iPSC-derived NSC-like cells for spinal cord injury is registered yet, a recent report describes preparation of clinical grade cells for this purpose. ${ }^{[23]}$

In Parkinson's disease, a neurodegenerative disorder resulting from progressive loss of dopaminergic neurons, application of NSCs with an acquired specification for a dopaminergic neuronal identity has demonstrated promising results. Dopaminergic neural progenitor cells can either be isolated from the fetal ventral midbrain ${ }^{[24]}$ or generated in vitro from human ESCs or iPSCs. ${ }^{[25]}$ Preclinical data show that transplantation of dopaminergic progenitors in rat and monkey Parkinson's disease models induced behavioural recovery. ${ }^{[14,16,26]}$ However, the initial proof-of-principle clinical studies using NSCs harvested from fetal ventral midbrains showed mixed results, ranging from no clinical benefit (even with demonstrated long-term engraftment) to excessive and uncontrolled dopamine production and major dyskinesia. ${ }^{[27]}$ In this context, we identified 4 ongoing registered clinical trials for Parkinson's disease, each using NSCs derived from different sources, namely fetal, ESCs, iPSCs and oocytes. The studies using fetal and ESC-derived NSCs were both initiated in 2017 (NCT03128450, NCT03119636), for which no data have yet been reported, while the study using iPSC-derived NSCs is planned for initiation in 2019 (NCT03815071). In addition to the latter, and not listed in clinicaltrials.gov, a clinical study using NSCs derived from iPSCs was initiated in 2018 in Japan. ${ }^{[16]}$ In the single 
study using oocytes as a source (NCT02452723), NSC-like cells were differentiated from human parthenogenetic stem cells obtained from chemically activated unfertilised oocytes. ${ }^{[28]}$ These cells are being used to treat patients with Parkinson's disease, with preliminary data showing safety with indications of efficacy over an ongoing 5-year period of follow-up. ${ }^{[29]}$

For treatment of brain tumours, 3 registered clinical studies use fetal-derived NSCs which have been gene modified to express cytosine deaminase, an enzyme involved in the activation of chemotherapeutic agents such as 5-fluorouracil and irinotecan, which serves to localise drug activity to the site of transplantation. Data from one of these studies have been reported, wherein 15 patients were recruited and proof-of-principle for this approach demonstrated ${ }^{[30]} \mathrm{A}$ fourth ongoing study applies fetal-derived NSCs loaded with an oncolytic virus to enhance tumour eradication in patients with glioblastoma (NCT03072134). Promising preclinical evidence using this approach suggests efficacy without toxicity. ${ }^{[2,31]}$

\section{Haematopoietic stem cells}

Haematopoietic stem cells are multipotent stem cells traditionally used for the treatment of malignant and non-malignant diseases of the blood and immune systems. They can be harvested directly from bone marrow, peripheral blood (following pre-treatment to mobilise HSCs from the bone marrow) or umbilical cord blood. Depending on the condition being treated, HSCs are either collected from the patient or from a healthy donor to be used for autologous or allogeneic transplantation, respectively. Allogeneic transplantation, however, comes with the constraint of having to genetically match the donor and recipient in order to avoid graft rejection or, more concerningly, the risk of graft v. host disease and its associated mortality. Patients are also subjected to lifelong immunosuppressive therapy.

HSC transplantation is performed in over 80 countries and, of the more than 60000 HSC transplants that are done globally per annum, the vast majority $(\sim 90 \%)$ are for treating haematological malignancies, including leukaemia, lymphoma and myeloma. ${ }^{[32,33]}$ Secondary to these indications is the treatment of solid tumours, while non-malignant conditions include bone marrow failure, haemoglobinopathies and primary immune disorders. In all of these cases, the underlying principle of HSC transplantation is to replenish the bone marrow with stem cells which engraft and reconstitute the immune system with a functional lineage of haematopoietic cells.

In addition to the above traditional indications, there has over the last decade been a distinct increase in the use of HSCs for regenerative purposes, particularly umbilical cord blood (UCB) as a source of HSCs for treating neurological conditions. ${ }^{[7]}$ In fact, our findings show that of the 54 clinical studies registered to treat neurological disease with HSCs, 40 use UCB as a source. Once harvested, an UCB unit is processed to separate mononuclear cells (via removal of the red blood cells and plasma), which ultimately comprises a high proportion of HSCs, and to a lesser extent MSCs, endothelial progenitor cells and immunosuppressive cells such as regulatory $\mathrm{T}$ cells and monocyte-derived suppressor cells. Altogether and once transplanted, these cells have been shown to provide a paracrine effect that (i) promotes cell survival; (ii) stimulates proliferation and migration of NSCs; (iii) induces regeneration of damaged brain cells; (iv) reduces inflammation; and $(v)$ promotes angiogenesis. ${ }^{[34]}$

Cerebral palsy and hypoxic ischaemic encephalopathy (HIE) are the neurological indications most treated with UCB on an experimental basis, with 17 and 10 registered clinical studies (out of 40), respectively. HIE is a significant risk factor for developing cerebral palsy and occurs during birth as a result of reduced blood flow to the brain. Up to $20 \%$ of these cases result in death of the newborn, and nearly $30 \%$ develop permanent neurological abnormalities. To our knowledge, only 1 of the 10 registered experimental clinical studies using UCB for HIE (NCT00593242) has reported data. ${ }^{[35]}$ In this pilot study with 23 participants, the authors demonstrate that the collection, preparation and infusion of fresh (non-cryopreserved) UCB cells into newborns with HIE is a feasible and safe approach, but requires a well-orchestrated multidisciplinary collaboration at the treating centre. Promising indicators of benefit at a 12-month follow-up were also reported. Subsequently, a larger randomised clinical study was registered in 2017, which aims to recruit 160 study participants (NCT02612155).

Several studies have been reported for the use of UCB to treat cerebral palsy. In the first, in which 140 children with cerebral palsy were treated with autologous UCB, safety and feasibility was demonstrated, but no efficacy data were reported, primarily owing to substantial differences in the quality of the harvested UCB units. ${ }^{[36]}$ The same group initiated a placebo-controlled randomised trial (NCT01147653) and reported a significant improvement in motor function and brain connectivity, but only in study participants who received higher cell doses ( $>20$ million cells $/ \mathrm{kg}$ ), reiterating the need to ensure that UCB is harvested and processed appropriately. ${ }^{[37]}$ These findings confirm those from a previously reported randomised trial from another group, in which the UCB cell dose was positively correlated with improved motor outcomes. ${ }^{[38]}$ In another randomised study, this time using allogeneic UCB together with recombinant human erythropoietin, a significant improvement in cognitive and motor function was reported relative to the control group ${ }^{[39]}$ Finally, Novak et al. ${ }^{[40]}$ performed a meta-analysis of all cell therapies used to treat cerebral palsy (olfactory ensheathing cells, NSCs and neural progenitor cells, and allogeneic UCB), from which it was shown that UCB seemed to be most effective means of treatment. One study was initiated in 2009 using peripheral blood mobilised HSCs to treat cerebral palsy (NCT01019733), with findings indicating that intrathecal injection of autologous HSCs was safe, but with limited improvement in neurological function after 6 months of follow-up. ${ }^{[41]}$

Autism spectrum disorder is another condition for which UCB is being explored. As the pathophysiology of the disorder includes aspects of neural inflammation and connectivity, the rationale for using UCB to provide paracrine benefits has been justified. ${ }^{[42]}$ Five clinical trials have been registered in this context, and study results have been reported for 2 (NCT01638819, NCT02176317). Both were Phase I/II clinical studies, which met the primary endpoints of safety and tolerability. In the first to be reported, 25 children were treated with autologous UCB infusions. ${ }^{[43]}$ Over a follow-up period of 6 months, significant improvements in behaviour were reported, including those with parent-reported measures and clinical assessments. ${ }^{[42,43]}$ A larger randomised trial has been registered (NCT02847182) to recruit 165 study participants. The second reported study was a placebo-controlled, randomised, cross-over design, wherein 30 young children were recruited. The authors reported an overall trend towards improvement, but with no statistically significant differences for any of the endpoints. ${ }^{[44]}$

Ten clinical studies using bone marrow-derived HSCs have been registered for the treatment of multiple sclerosis. Multiple sclerosis is to a large extent defined by the presence of autoreactive lymphocytes causing neural inflammation and degeneration. The goal of HSC transplantation is thus to reconstitute the immune system with a population of non-inflammatory cells. Recently, a preliminary communication reported data from a randomised clinical trial 
(NCT00273364) wherein 103 study participants received either HSC transplantation (non-myeloablative with low-dose chemotherapy) or disease-modifying therapy (typical treatment of multiple sclerosis). With a median follow-up of 2 years, disease progression occurred in 3 study participants who received HSC transplantation, while 34 receiving disease-modifying therapy progressed. ${ }^{[45]}$ A larger randomised Phase III study aiming to recruit 200 study participants has subsequently been registered by the same group and is ongoing (NCT03342638). All participants in this study will receive HSC transplantation and be randomised to study arms with differing conditioning regimens to maximise engraftment and patient outcomes.

\section{Mesenchymal stromal/stem cells}

Mesenchymal stem cells, also referred to as mesenchymal stromal cells or medicinal signalling cells, ${ }^{[46]}$ have the ability to self-renew and differentiate into cells of the mesoderm, including bone, adipose and cartilage. MSCs are believed to have high treatment potential based on several unique characteristics, including (i) their ability to home to a site of injury; (ii) their immunomodulatory and paracrine effects; (iii) the fact that they are immune-privileged (i.e. do not required genetic matching); and (iv) that they can be procured from many sources, such as bone marrow, adipose tissue and umbilical cord Wharton's jelly. MSCs from each of these sources are, however, more prone to differentiate into cells of their origin, and are hence more committed to these lineages.

To date, 3 MSC products have obtained regulatory approval for patient treatment. These include remestemcel-L (allogeneic bone marrow-derived MSCs) in Canada, New Zealand and Japan for acute graft-versus-host disease; darvadstrocel (allogeneic adiposederived MSCs) in Europe for fistulae in Crohn's disease; and stemirac (autologous bone marrow-derived MSCs) in Japan for treating spinal cord injury. None of these therapies has been approved for use in the USA, nor are they being used routinely in Europe as yet. With respect to the clinical trial landscape, MSCs are being applied to treat an extraordinary number of diseases - based on our investigations, as many as 150 different indications can be identified from registered clinical trials. Amongst these are a range of neurological conditions, diabetes, stroke, osteoarthritis, emphysema, bone fractures, wounds, macular degeneration and incontinence. Taken together, it is fair to say that MSCs are central to the stem cell hype as well as criticism of today, the latter being driven in part by the establishment of hundreds of unregulated clinics worldwide charging patients for a range of unproven treatments. ${ }^{[47,48]}$

Of the 120 registered clinical trials applying MSCs to treat neurological disease, nearly two-thirds are/were indicated for stroke $(n=27)$, spinal cord injury $(n=26)$ and multiple sclerosis $(n=23)$. In terms of tissue source, more than half of the registered trials use MSCs derived from bone marrow $(n=67)$, followed by adipose tissue $(n=26)$, umbilical cord $(n=23)$ and umbilical cord blood $(n=4)$. In each of these cases, the MSCs are expanded in cell culture to a therapeutic dose. With respect to the use of adipose tissue as a source, the treatment can either be in the form of stromal vascular fraction (SVF) or with culture-expanded MSCs, also referred to as adipose-derived stromal cells (ASCs). SVF is a concentrated extract of enzyme-digested adipose tissue which contains a mixture of ASCs, endothelial and endothelial precursor cells, lymphocytes, macrophages, smooth-muscle cells, pericytes and per-adipocytes. ${ }^{[4]}$ Similar to UCB, it is argued that this mixture of heterogeneous cells in SVF provides a broader therapeutic benefit. It is, however, limited for use as an autologous treatment (as it also contains white blood cells from the donor), but provides the advantage of being prepared and ready for infusion/transplantation within hours of the adipose tissue being harvested.

With 27 registered clinical studies, stroke is the most treated indication using MSCs. Sixteen use bone marrow as their source of MSCs, while 6 use adipose tissue (3 each apply SVF and expanded ASCs), 4 umbilical cord and 1 umbilical cord blood. Zheng et al. ${ }^{[50]}$ recently reviewed the literature regarding clinical reports relating to the use of MSCs for ischaemic stroke. They found 8 reported clinical studies, all of which were early-stage clinical trials, with only 3 being placebo-controlled. They conclude that there is a need for well-designed Phase II multicentre studies to definitively report the safety and preliminary efficacy of MSC treatment in patients with ischaemic stroke. In a more recent report of a 2-year follow-up of 16 patients receiving bone marrow-derived MSCs, it was shown that the treatment remained safe and was accompanied by encouraging improvements in clinical outcomes, particularly motor impairment scales. ${ }^{[51]}$ A larger placebo-controlled study has been initiated by the same group and is ongoing (NCT02448641).

Of the 26 clinical studies registered for spinal cord injury, 16 use MSCs expanded from bone marrow, 6 from adipose tissue and 4 from umbilical cord. In an excellent review published recently, ${ }^{[52]}$ it was indicated that 11 clinical studies have been reported to date where patients with spinal cord injuries were treated with either bone marrow- $(n=6)$ or umbilical cord-derived MSCs $(n=5)$. Cells were given either intrathecally and/or intravenously infused following expansion to various therapeutic doses. Taken together, these findings demonstrate that MSC administration in patients with spinal cord injury is safe and well tolerated (mild and temporary sideeffects in some cases). However, relative to rehabilitation therapy, no significant improvements in motor function were reported, although there were apparent and subtle improvements in sensory (light touch and pin prick) and bladder functions. Considering the dearth of treatment options and the morbidity associated with surgical intervention, one could argue that a stem cell-based therapy could be considered a less invasive alternative to alleviate the burden of this disease. However, large multicentre and randomised studies are required before this should be considered a viable option. ${ }^{[53]}$ As previously noted, 1 such treatment was recently granted approval by Japanese authorities, ${ }^{[4]}$ based on the treatment of 13 patients showing positive outcomes in a Phase I/II clinical trial. Notably, Japanese authorities do not require double-blinded randomised studies to grant approval for such therapies, which is a matter of ongoing debate, given that these studies are not placebo controlled and recruit only small numbers of patients.

For multiple sclerosis, the rationale for using MSCs is based on their immunomodulatory and neuroprotective properties. Based on our analysis of the 23 registered clinical trials for the disease, 15 employ MSCs derived from bone marrow, 4 umbilical cord and 4 adipose tissue ( 2 using SVF and 2 expanded MSCs). Although no pivotal clinical studies have been reported as yet, we identified numerous early-stage (Phase I/II) clinical reports employing bone marrow-derived MSCs. In a study reported in 2010, no adverse events and signs of clinical but not radiological efficacy were reported in 10 study participants following intravenous infusion. ${ }^{[54]}$ In a second study, 15 participants received intrathecal administration of autologous bone marrow-derived MSCs via lumbar puncture. The procedure was reported to be feasible and to have an acceptable safety profile, while it also showed signs of clinical stabilisation or improvement in some patients. ${ }^{[55]}$ Third, 10 study participants were treated with autologous bone marrow-derived MSCs in a 
Phase IIb study, ${ }^{[56]}$ where it was shown that MSCs provided benefits suggestive of neuroprotection based on their immunomodulatory and anti-inflammatory properties. The most recent report using bone marrow-derived MSCs for multiple sclerosis also included follow-up administrations of MSC-conditioned medium. ${ }^{[57]}$ MSC-conditioned medium, i.e. the same cell culture medium used to expand MSCs, contains a range of cytokines, chemokines and growth factors which are postulated to further promote neuronal regeneration. In this clinical study with 10 participants, the procedure was shown to be well tolerated with relative efficacy in stabilising the disease and reversing symptoms. Finally, we found 2 clinical reports of studies using ASCs for multiple sclerosis. In the first, 16 patients were treated by autologous, intrathecal administration of ASCs in a Phase I/II safety study. After 18 months of follow-up, ASCs were shown to be safe, but with no significant benefits having been reported. ${ }^{[58]}$ Notably, there was no disease progression, and the authors noted that although this was an attractive strategy, the treatment should be reserved for patients with aggressive disease progression. In the second study, a blinded randomised trial, 26 patients were treated with either low-dose/high-dose ASCs or placebo. No treatmentrelated adverse events were reported, along with an inconclusive trend regarding efficacy. ${ }^{[99]}$

\section{Summary and concluding remarks}

Neurological disorders are diverse and often poorly understood, for which there is generally a dearth of treatment options. Stem cell therapy holds great promise and may be a solution to alleviate the burden of these disorders. However, stem cells come in various 'shapes and sizes', and our lack of understanding of the basic biology and clinical benefits of transplanted stem cells currently prevents us from realising their true therapeutic potential. With over 200 registered clinical studies, progress is indeed being made. The vast majority of these are early-stage clinical trials designed primarily to assess safety and feasibility. Of the studies that have been reported, the general consensus is that stem cell therapy appears to be safe and well tolerated. Pivotal, well-designed randomised clinical trials are essential for measuring the true clinical benefits of these therapies.

The future of stem cell therapy for neurological disease is promising. We expect that more clinical studies using NSC-like treatments will be registered in the future, particularly those using iPSCs as a source of differentiated cells. It is apparent that UCB is becoming increasingly accepted as a feasible option for treating cerebral palsy and HIE. Data from a recent report for the use of non-myeloablative HSC transplantation in patients with multiple sclerosis are also encouraging. MSC-based treatments will remain attractive, given that they are a readily accessible resource and can be prepared with relative ease. However, therapeutic application of these cells should be based on rational premises. Early-stage clinical studies with promising indications of efficacy will progress into later-stage studies, and only those that show unequivocal efficacy in well-designed, randomised clinical trials will finally reach the market. As a cautionary note, complex disease requires complex therapies and, although public perception is that stem cell therapy could be a magic bullet for the cure of numerous ailments, it is unlikely to be administered as a monotherapy, but rather will form part of a more holistic approach that aims to significantly improve the quality of life of affected patients.

\section{Acknowledgments. None.}

Author contributions. MA: Conception, development of MSC clinical trial database, manuscript writing; OPS: Manuscript writing and editing;
KdB: Development of MSC clinical trial database; MSP: Conception, development of MSC clinical trial database, manuscript editing. Funding. Work in MSP's group is funded by the South African Medical Research Council (University Flagship and Extramural Unit awards).

\section{Conflicts of interest. None.}

1. Group GBDNDC. Global, regional, and national burden of neurological disorders during 1990-2015: systematic analysis for the Global Burden of Disease Study 2015. Lancet Neurol 2017;16(11):877-897. https://doi.org/10.1016/S1474-4422(17)30299-5

2. Gooch CL, Pracht E, Borenstein AR. The burden of neurological disease in the United States: A summary Gooch CL, Pracht E, Borenstein AR. The burden of neurol
report and call to action. Ann Neurol 2017;81(4):479-484

3. Pankevich DE, Altevogt BM, Dunlop J, Gage FH, Hyman SE. Improving and accelerating drug development for nervous system disorders. Neuron 2014;84(3):546-553. https://doi.org/10.1016/j. neuron.2014.10.007

4. Cyranoski D. Japan's approval of stem-cell treatment for spinal-cord injury concerns scientists. Nature 2019;565(7741):544-545. https://doi.org/10.1038/d41586-019-00178-x

5. Hoornaert CJ, Le Blon D, Quarta A, et al. Concise review: Innate and adaptive immune recognition of allogeneic and xenogeneic cell transplants in the central nervous system. Stem Cells Transl Med 2017;6(5):1434-1441. https://doi.org/10.1002/sctm.16-0434

6. Harris VK, Stark J, Vyshkina T, et al. Phase I trial of intrathecal mesenchymal stem cell-derived neural progenitors in progressive multiple sclerosis. EBioMedicine 2018;29:23-30. https://doi.org/10.1016/j. ebiom.2018.02.002

7. Dessels C, Alessandrini M, Pepper MS. Factors influencing the umbilical cord blood stem cell industry: An evolving treatment landscape. Stem Cells Transl Med 2018;7(9):643-650. https://doi.org/10.1002/ An evolving
sctm. $17-0244$

8. Grochowski C, Radzikowska E, Maciejewski R. Neural stem cell therapy - brief review. Clin Neurol Grochowski C, Radzikowska E, Maciejewski R. Neural stem cell therapy -

Neurosurg 2018;173:8-14. https://doi.org/10.1016/j.clineuro.2018.07.013
Christian KM, Song H, Ming GL. Functions and dysfunctions of adult hippocampal neurogenesis. Annu 9. Christian KM, Song H, Ming GL. Functions and dysfunctions of adult hippocampal neu
Rev Neurosci 2014;37:243-262. https://doi.org/10.1146/annurev-neuro-071013-014134

10. Sun GJ, Zhou Y, Stadel RP, et al. Tangential migration of neuronal precursors of glutamatergic neurons in the adult mammalian brain. Proc Natl Acad Sci USA 2015;112(30):9484-9489. https://doi.org/10.1073/ pnas. 1508545112

1. Kim HT, Kim IS, Lee IS, Lee JP, Snyder EY, Park KI. Human neurospheres derived from the fetal central nervous system are regionally and temporally specified but are not committed. Exp Neurol 2006;199(1):222-235. https://doi.org/10.1016/j.expneurol.2006.03.015

12. Vazin T, Chen J, Lee CT, Amable R, Freed WJ. Assessment of stromal-derived inducing activity in the generation of dopaminergic neurons from human embryonic stem cells. Stem Cells 2008;26(6):15171525. https://doi.org/10.1634/stemcells.2008-0039

13. Maden M. Retinoic acid in the development, regeneration and maintenance of the nervous system. Nat Rev Neurosci 2007;8(10):755-765. https://doi.org/10.1038/nrn2212

14. Kriks S, Shim JW, Piao J, et al. Dopamine neurons derived from human ES cells efficiently engraft in animal models of Parkinson's disease. Nature 2011;480(7378):547-551. https://doi.org/10.1038/
iniks S, Shim JW, Piao J, et al. in animal $\mathrm{m}$

15. Okita K, Ichisaka T, Yamanaka S. Generation of germline-competent induced pluripotent stem cells. Nature 2007;448(7151):313-317. https://doi.org/10.1038/nature05934

16. Stoddard-Bennett T, Reijo Pera R. Treatment of Parkinson's disease through personalized medicine and induced pluripotent stem cells. Cells 2019;8(1):26. https://doi.org/10.3390/cells8010026

17. Baker EW, Platt SR, Lau VW, et al. Induced pluripotent stem cell-derived neural stem cell therapy enhances recovery in an ischemic stroke pig model. Sci Rep 2017;7(1):10075. https://doi.org/10.1038/ s41598-017-10406-x

18. Ryu S, Lee SH, Kim SU, Yoon BW. Human neural stem cells promote proliferation of endogenous neural stem cells and enhance angiogenesis in ischemic rat brain. Neural Regen Res 2016;11(2):298-304. https:// doi.org/10.4103/1673-5374.177739

19. Tang Y, Wang J, Lin X, et al. Neural stem cell protects aged rat brain from ischemia-reperfusion injury through neurogenesis and angiogenesis. J Cereb Blood Flow Metab 2014;34(7):1138-1147. https://doi. through neurogenesis and

20. Sinden JD, Hicks C, Stroemer P, Vishnubhatla I, Corteling R. Human neural stem cell therapy for chronic Sinden JD, Hicks C, Stroemer P, Vishnubhatla I, Corteling R. Human neural stem cell therapy for chronic
ischemic stroke: Charting progress from laboratory to patients. Stem Cells Dev 2017;26(13):933-947. https://doi.org/10.1089/scd.2017.0009

21. Kalladka D, Sinden J, Pollock K, et al. Human neural stem cells in patients with chronic ischaemic stroke (PISCES): A phase 1, first-in-man study. Lancet 2016;388(10046):787-796. https://doi.org/10.1016/ S0140-6736(16)30513-X

22. Curtis E, Martin JR, Gabel B, et al. A first-in-human, phase I study of neural stem cell transplantation for chronic spinal cord injury. Cell Stem Cell 2018;22(6):941-950. https://doi.org/10.1016/j.stem.2018.05.014

23. Khazaei M, Ahuja CS, Rodgers CE, Chan P, Fehlings MG. Generation of definitive neural progenitor cells from human pluripotent stem cells for transplantation into spinal cord injury. Methods Mol Biol 2019;1919:25-41. https://doi.org/10.1007/978-1-4939-9007-8_3

24. Arenas E, Denham M, Villaescusa JC. How to make a midbrain dopaminergic neuron. Development 2015;142(11):1918-1936. https://doi.org/10.1242/dev.097394

25. Parmar M, Torper O, Drouin-Ouellet J. Cell-based therapy for Parkinson's disease: A journey through decades toward the light side of the Force. Eur J Neurosci 2018;49(4):463-471. https://doi.org/10.1111/ decades tow
ejn. 14109

26. Barker RA, Barrett J, Mason SL, Bjorklund A. Fetal dopaminergic transplantation trials and the future of neural grafting in Parkinson's disease. Lancet Neurol 2013;12(1):84-91. https://doi.org/10.1016/S14744422(12)70295-8

27. Lane EL, Bjorklund A, Dunnett SB, Winkler C. Neural grafting in Parkinson's disease unraveling the mechanisms underlying graft-induced dyskinesia. Prog Brain Res 2010;184:295-309. https://doi org/10.1016/S0079-6123(10)84015-4

28. Garitaonandia I, Gonzalez R, Sherman G, Semechkin A, Evans A, Kern R. Novel approach to stem cell therapy in Parkinson's disease. Stem Cells Dev 2018;27(14):951-957. https://doi.org/10.1089/ scd.2018.0001

29. Kern R, Garitaonandia I, Gonzalez R, Sherman G, et al. Interim clinical assessment of a neural stem cell based therapy for Parkinson's disease. Neurol 2018;90(15 Suppl): S26.002

30. Portnow J, Synold TW, Badie B, et al. Neural stem cell-based anticancer gene therapy: A first-in-human study in recurrent high-grade glioma patients. Clin Cancer Res 2017;23(12):2951-2960. https://doi. org/10.1158/1078-0432.CCR-16-1518

31. Ahmed AU, Thaci B, Tobias AL, et al. A preclinical evaluation of neural stem cell-based cell carrier for targeted antiglioma oncolytic virotherapy. J Natl Cancer Inst 2013;105(13):968-977. https://doi. org/10.1093/jnci/djt141

32. Passweg JR, Baldomero H, Bader P, et al. Is the use of unrelated donor transplantation leveling off in Europe? The 2016 European Society for Blood and Marrow Transplant activity survey report. Bone Marrow Transplant 2018;53(9):1139-1148. https://doi.org/10.1038/s41409-018-0153-1 
33. Centre for International Blood \& Marrow Transplant Research. Current Uses and Outcomes of Hematopoietic Cell Transplantation (HCT): CIBMTR Summary Slides. 2017 http://www.cibmtr.org (accessed 10 December 2018).

34. McDonald CA, Fahey MC, Jenkin G, Miller SL. Umbilical cord blood cells for treatment of cerebral palsy: timing and treatment options. Pediatr Res 2018;83(1-2):333-344. https://doi.org/10.1038/pr.2017.236

35. Cotten CM, Murtha AP, Goldberg RN, et al. Feasibility of autologous cord blood cells for infant with hypoxic-ischemic encephalopathy. J Pediatr 2014;164(5):973-979. https://doi.org/10.1016/j. jpeds.2013.11.036

36. Sun J, Allison J, McLaughlin C, et al. Differences in quality between privately and publicly banked umbilical cord blood units: A pilot study of autologous cord blood infusion in children with acquired neurologic disorders. Transfusion 2010;50(9):1980-1987. https://doi.org/10.1111/j.1537-2995.2010.02720.x

37. Sun JM, Song AW, Case LE, et al. Effect of autologous cord blood infusion on motor function and brain connectivity in young children with cerebral palsy: A randomized, placebo-controlled trial. Stem Cells Transl Med 2017;6(12):2071-2078. https://doi.org/10.1002/sctm.17-0102

38. Kang M, Min K, Jang J, et al. Involvement of immune responses in the efficacy of cord blood cell therapy for cerebral palsy. Stem Cells Dev 2015;24(19):2259-2268. https://doi.org/10.1089/scd.2015.0074

39. Min K, Song J, Kang JY, et al. Umbilical cord blood therapy potentiated with erythropoietin for children with cerebral palsy: A double-blind, randomized, placebo-controlled trial. Stem Cells 2013;31(3):581 591. https://doi.org/10.1002/stem.1304

40. Novak I, Walker K, Hunt RW, Wallace EM, Fahey M, Badawi N. Concise review: Stem cell interventions for people with cerebral palsy: Systematic review with meta-analysis. Stem Cells Trans Med 2016;5(8):1014-1025. https://doi.org/10.5966/sctm.2015-0372

1. Mancias-Guerra C, Marroquin-Escamilla AR, Gonzalez-Llano O, et al. Safety and tolerability of intrathecal delivery of autologous bone marrow nucleated cells in children with cerebral palsy: A open-label phase I trial. Cytotherapy 2014;16(6):810-820. https://doi.org/10.1016/j.jcyt.2014.01.008

42. Carpenter KLH, Major S, Tallman C, et al. White matter tract changes associated with clinica improvement in an open-label trial assessing autologous umbilical cord blood for treatment of youn children with autism. Stem Cells Transl Med 2019;8(2):138-147. https://doi.org/10.1002/sctm.18-0251

43. Dawson G, Sun IM, Davlantis KS, et al. Autologous cord blood infusions are safe and feasible in youn children with autism spectrum disorder: Results of a single-center phase I open-label trial. Stem Cells Transl Med 2017;6(5):1332-1339. https:// doi.org/10.1002/sctm.16-0474

44. Chez M, Lepage C, Parise C, Dang-Chu A, Hankins A, Carroll M. Safety and observations from placebo-controlled, crossover study to assess use of autologous umbilical cord blood stem cells to placebo-controlled, crossover study to assess use of autologous umbilical cord blood stem cells to
improve symptoms in children with autism. Stem Cells Transl Med 2018:7(4):333-341. https://doi. improve symptoms in chil

45. Burt RK, Balabanov R, Burman J, et al. Effect of nonmyeloablative hematopoietic stem cell transplantation vs continued disease-modifying therapy on disease progression in patients with relapsing-remitting multiple sclerosis: A randomized clinical trial. JAMA 2019;321(2):165-174. https:/ doi.org/10.1001/jama.2018.1874
46. Caplan AI. Mesenchymal stem cells: Time to change the name! Stem Cells Transl Med 2017;6(6):14451451. https://doi.org/10.1002/sctm.17-0051

47. Galipeau J, Sensebe L. Mesenchymal stromal cells: Clinical challenges and therapeutic opportunities. Cell Stem Cell 2018;22(6):824-833. https://doi.org/10.1016/j.stem.2018.05.004

48. Turner L, Knoepfler P. Selling stem cells in the USA: Assessing the direct-to-consumer industry. Cell Stem Cell 2016;19(2):154-157. https://doi.org/10.1016/i.stem.2016.06.007

49. Bora P. Majumdar AS. Adipose tissue-derived stromal vascular fraction in regenerative medicine: A brief review on biology and translation. Stem Cell Res Ther 2017;8(1):145. https://doi.org/10.1186/ $13287-017-0598-1$

50. Zheng H, Zhang B, Chhatbar PY, et al. Mesenchymal stem cell therapy in stroke: A systematic review of literature in pre-clinical and clinical research. Cell Transplant 2018;27(12):1723-1730. https://doi. rg/10.1177/0963689718806846

51. Steinberg GK, Kondziolka D, Wechsler LR, et al. Two-year safety and clinical outcomes in chronic ischemic stroke patients after implantation of modified bone marrow-derived mesenchymal stem cells (SB623): A phase 1/2a study. J Neurosurg 2018:1-11. https://doi.org/10.3171/2018.5.JNS173147

52. Xu P, Yang X. The efficacy and safety of mesenchymal stem cell transplantation for spinal cord injury patients: A meta-analysis and systematic review. Cell Transplant 2018;28(1):36-46. https://doi. org $/ 10.1177 / 0963689718808471$

53. Khan S, Mafi P, Mafi R, Khan W. A systematic review of mesenchymal stem cells in spinal cord injury, intervertebral disc repair and spinal fusion. Curr Stem Cell Res Ther 2018;13(4):316-323. https://doi.or g/10.2174/1574888X11666170907120030

54. Yamout B, Hourani R, Salti H, et al. Bone marrow mesenchymal stem cell transplantation in patients with multiple sclerosis: A pilot study. J Neuroimmunol 2010;227(1-2):185-189. https://doi org/10.1016/j.jneuroim.2010.07.013

55. Karussis D, Karageorgiou C, Vaknin-Dembinsky A, et al. Safety and immunological effects of mesenchymal stem cell transplantation in patients with multiple sclerosis and amyotrophic lateral sclerosis. Arch Neurol 2010:67(10):1187-1194. https//doi.org/10.1001/archneurol.2010.248

56. Connick P, Kolappan M, Crawley C, et al. Autologous mesenchymal stem cells for the treatment of secondary progressive multiple sclerosis: An open-label phase 2a proof-of-concept study. Lancet secondary progressive multiple sclerosis: An open-label phase 2a proof-c

57. Dahbour S, Jamali F, Alhattab D, et al. Mesenchymal stem cells and conditioned media in the treatment of multiple sclerosis patients: Clinical, ophthalmological and radiological assessments of safety and efficacy. CNS Neurosci Ther 2017;23(11):866-874. https://doi.org/10.1111/cns.12759

58. Stepien A, Dabrowska NL, Maciagowska M, et al. Clinical application of autologous adipose stem cells in patients with multiple sclerosis: Preliminary results. Mediators Inflamm 2016;2016:5302120. https:// doi.org/10.1155/2016/5302120

59. Fernandez O, Izquierdo G, Fernandez V, et al. Adipose-derived mesenchymal stem cells (AdMSC) for the treatment of secondary-progressive multiple sclerosis: A triple blinded, placebo controlled, randomized phase I/II safety and feasibility study. PLoS One 2018;13(5):e0195891. https://doi. org/10.1371/journal.pone.019589 